\title{
Zur Frage der Behandlung chronischer Alkoholisten.
}

\author{
Von \\ Dr. Fürer, \\ Haus Rockenau bei Eberbach (Baden).
}

Sehr bald, nachdem ich reichlicher Gelegenheit hatte, an chronischem Alkoholismus Leidende, vorwiegend der gebildeten Stände, zu behandeln, lrängte sich mir die Beobachtung auf, daß die meisten dieser Kranken in auffälliger Weise versagten, wenn man von ihnen das Festhalten an einer bestimmten, gewisse systematische Arbeit zu bestimmten Zeiten verlangenden Tageseinteilung verlangte. Auch die sonst intelligenten und einsichtigen Kranken, welche den übrigen von mir getroffenen Anordnungen ohne Schwierigkeiten folgten, versagten hier völlig. Während sie pünktlich zu den Bädern erschienen, nachher ihre Ruhezeiten innehielten, regelmäßig zur Massage, Elektrisieren usw. zur Stelle waren, die Mahlzeitsstunden beobachteten, Spaziergänge nicht ïber das erlaubte Maß ausilehnten usw., machten sie sofort Sohwierigkeiten, wenn sie regelmäßig zu gymmastischen Übungen ofler körperlicher Arbeit, sei es in Werkstätten oder im Freien sich einfinden sollten. Sie verfielen in ein nörglerisches Vergleichen mit dem, was anderen erlaubt wurde und ihnen nicht, wollten nicht einsehen, daß auch in ler Zuteilung aktive Anstrengung verlangender Arbeit der jeweilige /ustand der verschiedenen Kranken maßgebend sein müisse. Die Entgegnung: „Wenn der und der das nicht machen muß, brauche ich es doch auch nicht zu tun" "usw. wurde der AnlaB zu fruchtlosen Auseinandersetzungen und Verstimmungen, welche die übrige Behandlung erschwerten, mein sonst gutes Verhältnis zu den Kranken störten und meinen Einfluß auf sie in Frage zu stellen drohten. Daß ich mich an allen den verlangten körperlichen Arbeiten, soweit möglich beteiligte, änderte nichts an ihrem Verhalten. Vorïbergehend wohl konnte ich sie wieder zur Pïnktlichkeit in der Einhaltung der Beschäftigung veranlassen, aber sehr bald wieder stellte sich ihre Renitenz ein. Ich mulbte mich schließlich davon überzeugen, daß3 nicht Ubelwollen, sondern das Unvermögen zu einer auch in den mäßigsten Grenzen sich haltenden pflichtgemäßen aktiven Betätigung wohl die Veranlassung sei zu diesem (ler Intelligenz und dem sonstigen guten Willen der Kranken gar nicht entsprechenden Verhalten. Ich kam zu der Úberzeugung, daß das Nervensystem dieser Kranken zu konsequenter, zielbewußter Anstrengung weder körperlicher noch geistiger Art nicht fähig sei, und 
laß es sich bei ihnen um die Äußerung einer allgemeinen nervösen Erschöpfung und Erschöpfbarkeit handle, die erst überwunden sein müsse, bevor man mit der Erziehung zu systematischer Arbeit anfangen könne, daß diese Erziehung aber erst nach recht langer Zeit einsetzen, und daß3 mithin in den meisten Fällen der Aufenthalt in der Anstalt damit nicht belastet werden könne. Daß es für die Behandlung dieser Kranken sehr wichtig ist, ihnen keine Willensanstrengungen zuzumuten, welche über ihre Kräfte gehen, müßte eigentlich für Jeden einleuchtend sein, der sich mit ihrem psychischen Zustande vertraut gemacht hat. Je intelligenter die Kranken sind, um so mehr leiden sie darunter, daß ihr Wollen und Können nicht im richtigen Verhältnis zueinander stehen. Alles bei ihnen ist Vorsatz, dem stets die Enttäuschung des NichtausführenKönnens folgt, die um so deprimierender wirkt, je mehr die Kranken sich die Schuld an ihrem Nichtkönnen zuschreiben und je mehr Andere sie in dieser Idee bestärken. Die Vorstellung, daß es nur einer ihnen möglichen Willensanstrengung bedïrfe, um ihnen aus der Misere ihres Zustandes zu helfen, bringt sie der Verzweiflung nahe. Die Behandlung hat $m$. E. an diesem Punkte anzugreifen und den Kranken begreiflich $\%$ machen, daß es sich in erster Linie darum handeln muß, die zu zielbewußter Energieentwicklung notwendige Gesundheit des Nervensystenss ihnen wieder zu verschaffen.

Zur Stütze meiner Anschauung über die Schonungsbedürftigkeit dieser Kranken, die ich von da ab meiner Behandlung zugrunde legte, war ich auf mehr subjektive Beobachtungen angewiesen, welche für eine noch bestehende Störung des nervösen Gleichgewichtes sprachen. Solche waren: Verstimmbarkeit, Reizbarkeit, körperliche und geistige Ėrmüdbarkeit, übermäßig großes Schlaf- und Ruhebedürfnis, Schlafstörungen, Schwankungen im Verhalten des Körpergewichtes, funktionelle nervöse Störungen usw. Wenn auch diese Störungen im wesentlichen den bei einfachen funktionellen Neurosen zur Beobachtung kommenden entsprachen, so mußten sie doch meines Erachtens anders bewertet werden, da sie der Wirkung eines Giftes ihr Dasein verdankten, von dem wir wissen, daß es imstande ist, schwere anatomische Läsionen des Nervensystems hervorzurufen. Ich nahm nun an, daß es möglich sein müsse, den Beginn dieser Läsion in den Fällen festzustellen, welche zumeist in meine Behandlung kamen und das bekannte Bild des Stadiums der chronischen Alkoholintoxikation boten, welche sich bei meist geringfügigen und flüchtigen körperlichen Störungen vorwiegend in einer ausgesprochenen cerebralen Insuffizienz äußert. Mit fortschreitender Besserung des Zustandes unter geeigneten therapeutischen Maßnahmen, in erster Linie völliger Akoholabstinenz, mußte sich dann der etwa erhobene pathologische Befund bessern resp. verlieren.

Die Untersuchung der Cerebrospinalflüssigkeit schien mir hierzu 
geeignet, nachdem sich die Lumbalpunktion als ungefährlicher Eingriff erwiesen hatte und hinlänglich Untersuchungserfahrungen vorlagen.

Die Punktion wurde in 26 Fällen (männliche Kranke) 31 mal vorgenommen. In allen Fällen wurde auch Blut zur Untersuchung entnommen. Priv.-Doz. Dr. Ranke-Heidelberg hatte die Güte, die Untersuchungen zu machen. Der Eingriff verlief in den meisten Fällen symptomlos. Einige Male jedoch traten im Anschluß an denselben, einerlei ob er im Sitzen oder Liegen des Kranken vorgenommen wurde, bald nachher oder am folgenden Tag unangenehme subjektive Symptome, meist Kopfschmerzen, vage Schmerzempfindungen im ganzen Körper, Gefühle von Nackensteifigkeit, einmal lebhafteres Schwindelgefühl mit Erbrechen, einmal ein leichter Zustand von Verwirrtheit auf. Ich ließ die Kranken stets im Anschluß an die Punktion $2 \times 24$ Stunden in möglichst horizontaler Lage im Bett liegen, resp. so lange, bis die subjektiven Beschwerden verschwunden waren.

In der folgenden Tabelle sind alle untersuchten Fälle angeführt. Das Pluszeichen bedeutet einen pathologischen, das Minuszeichen einen normalen Befund. Ein Fragezeichen bei Plus- oder Minuszeichen einen eher pathologischen als normalen Befund, resp. umgekehrt; ein Fragezeichen allein einen unsicheren, jedoch wohl nicht normalen Befund. Ein Eiweilggehalt bis 2,5 Esbach (nach $\mathrm{Nissl}$ ) gilt noch als normal, ein solcher von $2,5-3,0$ als leicht, darüber hinaus als stark pathologisch. Jede Zellenvermehrung gilt als pathologiseh.

\begin{tabular}{|c|c|c|c|c|c|c|c|c|c|c|}
\hline 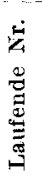 & $\begin{array}{l}\text { 离 } \\
\text { 湈 }\end{array}$ & 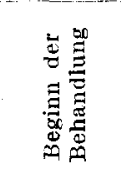 & 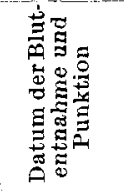 & 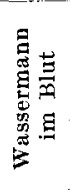 & 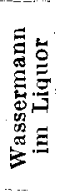 & $\begin{array}{l}\text { Zellen im } \\
\text { Liquor }\end{array}$ & 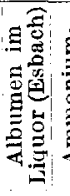 & 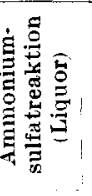 & $\begin{array}{l}\text { Goldsol- } \\
\text { reaktion } \\
\text { Jiquor }\end{array}$ & $\begin{array}{l}\text { Lues. } \\
\text { Infektions- } \\
\text { jahr }\end{array}$ \\
\hline 1 & $39 \mathrm{~J}$. & 3. I. 12 & 9. I. 12 & - & - & - & 2,0 & - & & - \\
\hline 2 & $25 \mathrm{~J}$. & 29. X. 12 & 31. X. 12 & - & - & - & 2,1 & - & - & - \\
\hline 3 & $32 \mathrm{~J}$ & |2.VII. 12 & 24.VII. 12， & - & - & - & 2,0 & - & & - \\
\hline 4 & $27 \mathrm{~J}$ & 19.VI.12 & 25. VI. 12 & .. & - & - & 2,11 & -.. & & - \\
\hline 5 & $30 \mathrm{~J} .1$ & 12.VI.1t & 21). VI. 11 & - & - & - & 2,4 & - & & - \\
\hline 6 & $49 \mathrm{~J}$ & 3.XII. 12 & 7. II. 11 & - & - & - & 6,0 & - & & - \\
\hline " & 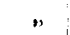 & $"$ & $18, v .11$ & - & - & - & 2,5 & - & & - \\
\hline 7 & $47 \mathrm{~J}$. & 30. XI. 11 & 4. XII. 11 & - & - & leicht vermehrt & 3,2 & - & & - \\
\hline$"$ & $"$ & ; & 5. 11. 12 & - & - & - & 2,0 & - & & - \\
\hline 8 & $31 \mathrm{~J}$. & 3. XII. 11 & 19.XII.11 & - & - & deutl. vermehrt & 3,5 & - & & - \\
\hline 9 & $39 \mathrm{~J}$ & 3. I. 12 & 9.1.12! & - & - : & leicht vermebrt & 2,6 & - & & - \\
\hline 10 & ד 39 & 16.1 .12 & 22. I. 12 & - & - & leicht vermehrt & 2,8 & - & & - \\
\hline , & $"$ & $”$ & 11. IV. 12 & - & - & reichl. vermehrt & 1,8 & - & & - \\
\hline 11 & $25 \mathrm{~J}$ & $24 . \times 12$ & 30. X. 12 & - & - & 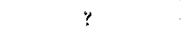 & 4,0 & - & - & - \\
\hline 12 & $44 \mathrm{~J}$. & 16. III. 12 & 18. III. 12 & - & - : & leicht vermehrt & 2,5 & - & & - \\
\hline 13 & $31 \mathrm{~J}$ & 22. XI. 11 & 28. XI. 11 & - & - & leicht vermehrt & 1,2 & - & & - \\
\hline 14 & $39 \mathrm{~J}$. & 16. XI. 12 & $22 . \times 1.12$ & - & - & $?$ & 4,5 & - & minimal & - \\
\hline 15 & $40 \mathrm{~J}$. & 8. XI. 12 & 22. XI. 12 & - & - & - & 4,8 & - & minimal & - \\
\hline
\end{tabular}




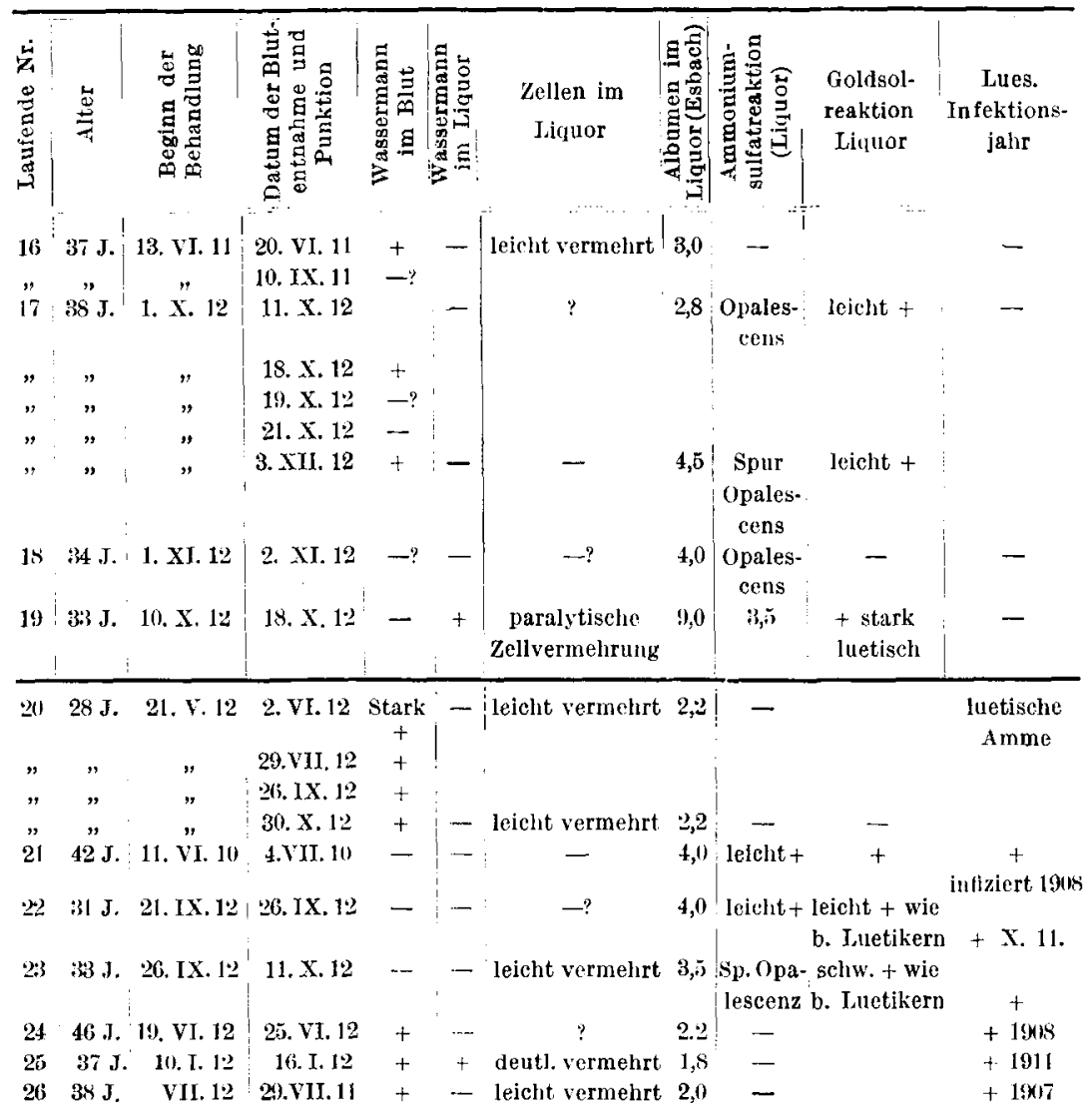

Die ersten 15 Fälle repräsentieren die reinen Fälle von chronischem Alkoholismus. Von den ersten 5 Fällen ohne pathologischen Befund scheiden Fall 1 und 2 aus. Sie betreffen Kranke, welche (Fall 1) früher an chronischem Alkoholismus leidend, dann nach 7 jähriger vollständiger Alkoholabstinenz seit kurzem rezidiviert waren, resp. (Fall 2) schon seit etwa 1 Jahr nur noch selten alkoholische Exzesse begangen hatten. Bei beiden bestand in der Hauptsache eine verminderte Leistungsfähigkeit mit hypochondrisch neurasthenischen Beschwerden, resp. neurasthenischer Haltlosigkeit. Im ersten Falle wäre anzunehmen, daß das kurzdauernde Rezidiv noch nicht zu Veränderungen geführt, im anderen, daß etwa vorhanden gewesene Veränderungen in der Zeit geringeren Alkoholgenusses sich zurückgebildet hätten. Fall 3 betraf einen Kranken mit typischem, seit Jahren bestehenden chronischen Alkoholismus ohne körperlichen Befund, Fall 4 und 5 solche mit langjährigem starkem Alkoholismus auf Grund ausgesprochen psychopathischer Veranlagung. deren Beschwerden rein neurasthenischen Charakter trugen. In den 
folgenden 10 Fïllen $(6-15)$ handelt es sich um reine Fälle typischen Alkoholismus' ohne Organerkrankungen schwerer Art. Die meisten von ihnen zeigen eine z. T. sehr starke Eiweißvermehrung, kombiniert mit Zellvermehrung. Besondere Wichtigkeit möchte ich den Fällen 6, 7, 10 beimessen, in welchen die einige Monate später vorgenommene Nachuntersuchung eine weitgehende Besserung, im Fall 7 völlig normale Verhältnisse konstatierte. - Es fanden sich also unter 13 Fällen von typischem chronischem Alkoholismus 10 Fälle mit positiv pathologischem Befunde $(77 \%)$, von welchen die Fälle 6, 7 und 10 im Verlaufe der Behandlung eine weitgehende Besserung erfuhren. Diese Konstatierung ist meines Erachtens geeignet, eine Stütze für meine oben entwickelte Anschauung von der Schonungsbedürftigkeit des Nervensystems dieser Kranken zu bilden. Auf alle Fälle aber sollte sie dazu anregen, gleiche Untersuchungen vorzunehmen. In der vorhandenen Literatur sind derartige Befunde meines Wissens bisher noch nicht mitgeteilt.

Was nun die Fälle 16-19 anlangt, so stellten alle diese Kranken eine luetische Erkrankung auf das bestimmteste in Abrede. Sie hatten nic eine antiluetische Behandlung durchgemacht. Schien es schon auffällig, daß im Fall 16 ohne spezifische Behandlung der Blutbefund wahrscheinlich negativ wurde im Verlaufe einiger Monate, so ist der Fall 17 wohl geeignet, zur allergrößten Vorsicht in der Verwertung einmaliger Blutbefunde zu mahnen. Fall 18 und 19 nehmen vielleicht insofern noch eine Sonderstellung ein, als Fall 18 vor 20 Jahren eine schwere Diphtherie mit längerdauernder Lähmung aller Fxtremitäten und Fall 19 vor 23 Jahren eine solche mit Otitis media dextra und linksseitiger Lühmung durchmachten. Residuen dieser Lähmungen bestehen nicht. In beiden Fällen spricht nichts sonst für eine überstandene Lues.

Wenn man diese Fülle noch zu den beweiskräftigen Fällen rechnet, so würde bei 14 von 17 Fällen chronischen Alkoholismus' der Beweis ('iner nachweisbaren Läsion des Zentralnervensystems erbracht sein, d. h. bei $82 \%$.

Von den 7 mit Lues komplizierten Fällen nimmt Fall 20 insofern eine Sonderstellung ein, als es sich um einen Alkoholisten handelt, wel(her eine notorisch luetische Amme gehabt hatte, aber nie selbst Symptome der Lues geboten hatte. Interessant ist bei ihm, daß im Laufe von fast 5 Monaten ohne spezifische Behandlung lediglich der Blutbefund sich gebessert hatte. Ob die 'Tatsache, daß alle luetischen Alkoholiker einen pathologisehen Befund boten, eine Verwertung in der Richtung erlaubt, daß die Kombination von Alkoholintoxikation und Lues für das Zentralnervensystem besonders verderblich ist, wofür ja viele andersartige Beobachtungen sprechen, muß bei der geringen Zahl der Fälle wohl dahingestcllt bleiben. 